\title{
Inefficient purifying selection: the mammalian $Y$ chromosome in the rodent genus Mus
}

\author{
Sara A. Sandstedt, Priscilla K. Tucker \\ Department of Ecology and Evolutionary Biology, and Museum of Zoology, University of Michigan, Ann Arbor, Michigan 48109, USA
}

Received: 1 April 2005 / Accepted: 8 September 2005

\begin{abstract}
Two related genes with potentially similar functions, one on the $\mathrm{Y}$ chromosome and one on the $\mathrm{X}$ chromosome, were examined to determine if they evolved differently because of their chromosomal positions. Six hundred fifty-seven base pairs of coding sequence of Jarid1d (Smcy) on the Y chromosome and Jarid1c (Smcx) on the $\mathrm{X}$ chromosome were sequenced in 13 rodent taxa. An analysis of replacement and silent substitutions, using a counting method designed for samples with small evolutionary distances, showed a significant difference between the two genes. The different patterns of replacement and silent substitutions within Jarid1d and Jarid1c may be a result of evolutionary mechanisms that are particularly strong on the $\mathrm{Y}$ chromosome because of its unique properties. These findings are similar to results of previous studies of $Y$ chromosomal genes in these and other mammalian taxa, suggesting that genes on the mammalian $\mathrm{Y}$ evolve in a chromosome-specific manner.
\end{abstract}

\section{Introduction}

Chromosomal sex determination has evolved separately in some plants, fishes, birds, insects, and in mammals (reviewed by Bull 1983). Interestingly, the properties of $\mathrm{Y}$ chromosomes are similar in some of the independently evolved instances that have been studied (e.g., Fridolfsson et al. 1998; Steinemann and Steinemann 1998; Tucker et al. 2003; Ceplitis and Ellegren 2004; Zhang 2004). One model of sex

\footnotetext{
${ }^{*}$ Present address: Department of Epidemiology, University of Michigan, 109 Observatory, Rm 2010 School of Public Health Building I, Ann Arbor, MI 48109, USA

Correspondence to: Sara A. Sandstedt; E-mail: sandsted@umich. edu
}

chromosome evolution, derived mainly from studies in Drosophila, is that chromosomal sex determination may evolve from genic sex determination by the gradual accumulation of sex-specific genes in the region of the sex-determining gene, where they would be more likely to be inherited by offspring of the correct sex. Suppression of recombination may keep these genes that are beneficial to males from being inherited by females but also allow divergence and erosion of the sex-specific genes (reviewed by Rice 1996).

While much is known about the evolution of the $\mathrm{Y}$ in Drosophila, less is known about other organisms. For example, the dioecious plant Silene latifolia appears to differ from Drosophila and mammals because its $\mathrm{Y}$ chromosome may not have experienced degeneration and its $\mathrm{X}$ lacks dosage compensation (reviewed by Negrutiu et al. 2001). Because independently evolved Y chromosomes may behave differently from the model, it is useful to study the evolution of the Y chromosome in depth in other organisms, including mammals.

Distinguishable $\mathrm{X}$ and $\mathrm{Y}$ chromosomes that evolved from a homologous chromosome pair are found in all mammalian groups including eutherians (placental mammals), monotremes, and marsupials (reviewed by Toder et al. 2000). Sex chromosomes have characteristics that may cause their genes to evolve with different rates and patterns from genes on other chromosomes. Two of these characteristics are that most of the $\mathrm{Y}$ is completely genetically linked and it has a small effective population size, each of which may interfere with natural selection through several mechanisms (recently reviewed by Rice 1996; Charlesworth and Charlesworth 2000; Lahn et al. 2001; Tucker et al. 2003). We are interested in determining if genes on the $\mathrm{Y}$ chromosome show patterns of evolution consistent with an interference with natural selection, which causes purifying selection to be inefficient, or if these genes 
evolve in a locus-specific manner related to their particular functions.

If features of the $\mathrm{Y}$ chromosome interfere with purifying selection, the ratio of nonsynonymous changes per nonsynonymous site to synonymous changes per synonymous site $(\mathrm{dN} / \mathrm{dS})$ should be less than one, but more than the baseline $\mathrm{dN} / \mathrm{dS}$ ratio caused by purifying selection. The gene under study may be compared to a similar gene that is thought to undergo purifying selection. Some Y chromosomal genes have "gametologs" which are related genes found on the $\mathrm{X}$ chromosome, with similarity retained from before recombination between the $\mathrm{X}$ and Y chromosomes ceased (Garcia-Moreno and Mindell 2000). If purifying selection is interfered with by the properties of the $\mathrm{Y}$, the $\mathrm{dN} / \mathrm{dS}$ of a $\mathrm{Y}$ chromosomal gene is expected to be higher than the $\mathrm{dN} / \mathrm{dS}$ of its $\mathrm{X}$ chromosomal gametolog.

Recently, pairwise comparisons of gametologs were made between human and mouse, and human and macaque (Wyckoff et al. 2002). Indeed, the dN/ $\mathrm{dS}$ of the Y chromosomal genes was higher than the $\mathrm{dN} / \mathrm{dS}$ of their $\mathrm{X}$ chromosomal gametologs. It was concluded that either the $\mathrm{Y}$ chromosome genes are continuing to evolve new functions or that a $\mathrm{Y}$ chromosomal effect is causing more nonsynonymous substitutions. These results are intriguing, but it is also valuable to examine this question in a finerscale phylogenetic context in which less time has elapsed and fewer multiple hits have occurred. We chose to examine gametologous gene pairs within the mouse genus Mus. One advantage of using this particular system is that two Y chromosomal genes (Sry and Zfy) have already been examined in these species. However, each of these genes may have evolved as it did because of factors unrelated to their positions on the $\mathrm{Y}$ chromosome (Tucker and Lundrigan 1993; Jansa et al. 2003; Tucker et al. 2003), compelling further study.

Here we examine gametologs that are not as likely to be affected by complicating factors. We sequenced and analyzed Jarid1d from the Y chromosome [formerly Smcy, Selected mouse cDNA on $\underline{\mathrm{Y}}$ (Agulnik et al. 1994a)] and Jarid $\overline{1 c}$ from the X chromosome [formerly Smcx (Agulnik et al. 1994b)] in species of the mouse genus Mus (family Muridae, subfamily Murinae). These genes are members of the highly conserved Jarid subfamily (reviewed by Patsialou et al. 2005) and were renamed to reflect that relationship in January 2004 (http://www.informatics.jax.org). Jarid1c partially escapes $\mathrm{X}$ inactivation in mice (Wu et al. 1994b; Sheardown et al. 1996) and in humans (Wu et al. 1994a). Jarid1c also appears to escape $\mathrm{X}$ inactivation in other primates, horses, dogs, whales, and elephants (Jegalian and Page 1998), al- though more Jarid1c mRNA is expressed in female mouse brains than Jarid1c and Jarid1d combined in male mouse brains (Xu et al. 2002). In gametolog pairs in which the X-linked gene escapes inactivation, males may be constrained to have exactly one functionally equivalent gene on the $\mathrm{Y}$ chromosome to maintain an equivalent gene dosage (reviewed by Lahn and Page 1997; Disteche 1999). Jarid1d and Jarid1c were chosen for this study because the genes are similar in expression patterns (Agulnik et al. 1994a) and seem likely to share functions (Agulnik et al. 1999; but see Jensen et al. 2005). Thus, differences between them are more likely to be due to their chromosomal positions than differences between other gene pairs that have very different expression patterns or functions. We report a Y chromosomal effect on the evolution of Jarid1d when compared to Jarid $1 c$ in the mammalian genus Mus.

\section{Materials and methods}

Species. Thirteen taxa, including some of the most commonly studied species of the genus Mus, were used (see Lundrigan et al. 2002 for collecting information). The genera Mus, Mastomys, and Hylomyscus, are members of the rodent family Muridae and the subfamily Murinae, the Old-world Mice and Rats. Many of the same species used in the study of rodent Sry (Tucker and Lundrigan 1993) were used here to make the results more comparable. The sister group to Mus is unknown, although there is evidence from DNA hybridization and nuclear DNA sequence for a sister group relationship with the Praomys group (Catzeflis and Denys 1992; Chevret, et al. 1994; Jansa and Weksler 2004). Members of the Praomys group (Hylomyscus alleni and Mastomys hildebrandtii) were used as outgroups in rooted trees in this study.

Amplification of the target sequences. In mice, Jarid1d and Jarid1c share the same genomic structure of 26 exons (Agulnik et al. 1999). Jarid1d is present in marsupials, indicating that Jarid1c and Jarid1d diverged at least 120 million years ago (Agulnik et al. 1994a). Jarid1d encodes several of the H$\mathrm{Y}$ antigens that can cause the rejection of male-donor tissue transplants in female recipients (Scott et al. 1995; Wang et al. 1995). Both Jarid1d and Jarid1c are members of the jumonji family of transcription factors (Takeuchi et al. 1995) and Jarid1c mutations are related to X-linked mental retardation in humans (Jensen et al. 2005).

The polymerase chain reaction (PCR) (Saiki et al. 1985) was used as described, with primers developed 
specifically for these species (Sandstedt and Tucker 2004), to amplify a fragment that includes exons 23 and 24, a portion of exon 25, and introns 23,24 and 25 from both genes in all taxa. All sequences were deposited in GenBank (accession No. \#AY260478AY260503).

\section{Analysis}

Phylogeny. Jarid1d and Jarid1c nucleotide sequences were aligned using Clustal X (Thompson et al. 1997) and adjusted by hand using amino acid translations as a guide with the program MacClade 4.0 (Maddison and Maddison 2000). Unambiguous alignments of exon sequences were obtained for each gene separately using Clustal X (Thompson et al. 1997). Although there were $687 \mathrm{bp}$ in this region of Jarid1d and only 660 in Jarid1c, their comparison required the same length sequence for both genes. To produce equally long sequences, $\mathrm{X}$ and $\mathrm{Y}$ sequences were aligned together using Clustal $\mathrm{X}$. These alignments were adjusted by hand using a conceptual translation in MacClade as a guide. Characters that aligned with gaps were removed, totaling 30 bases from Jarid1d and 3 from Jarid1c. Maximum parsimony as implemented in PAUP ${ }^{\star}{ }^{\mathrm{v} 4.0 b 10}$ (Swofford 2002) was used to build unrooted gene trees from the Jarid1c and Jarid1d nucleotide sequences. The branch-and-bound option was used. Characters were equally weighted and unordered.Jarid1c and Jarid1d intron and exon data were also combined with sequences from four other nuclear genes in parsimony and maximum likelihood analyses as described (Tucker et al. 2005).

Silent and replacement substitutions. Because of the small number of substitutions in the sequences studied, a method that is particularly appropriate for small samples was used (Zhang et al. 1997). For this analysis, the uncorrected numbers of replacement and silent substitutions were counted along the branches of the most parsimonious nuclear tree, with its root removed. Ancestral sequences were reconstructed using the program Anc-Gene (Zhang and Nei 1997; Zhang et al. 1998; Zhang 2004). This program first determines the ancestral amino acid sequences using a distance-based Bayesian method, and then reconstructs the nucleotide sequence at each node. Both the Poisson model and the JTT empirical model of amino acid substitution under the extended parsimony mode of Anc-Gene were used. The resulting ancestral sequences were compared using the Nei and Gojobori method (Nei and Gojobori 1986) implemented in MEGA 2.1 (Kumar et al. 2001). Replacement and silent changes were mapped along each branch on the nuclear tree topology, separately for Jarid1c and Jarid1d. The numbers of replacement and silent changes per branch for each gene were added together, then compared using Fisher's exact test, which is appropriate for small samples, implemented in the Fisher program (Zhang et al. 1997). Similar analyses were done with parsimony ancestral reconstructions using the ACCTRAN and DELTRAN methods of ancestral reconstruction in PAUP ${ }^{\star} \mathrm{v} 4.0 \mathrm{~b} 10$ (Swofford 2002).

The number of potential sites for a replacement change, $\mathrm{N}$, and the number of potential sites for a silent change, S, were calculated using the Nei and Gojobori method (Nei and Gojobori 1986) implemented in MEGA 2.1 (Kumar et al. 2001). N and S were each averaged over the branches of the Jarid1d and Jarid $1 c$ trees, separately. The $\mathrm{dN} / \mathrm{dS}$ of each gene was calculated using the changes counted using Anc-gene and the averages of $\mathrm{N}$ and $\mathrm{S}$.

\section{Results}

Phylogenetic analyses. Eight unrooted equally most parsimonious trees were recovered from the branchand-bound search of 26 OTUs (13 taxa, Jarid1d and Jarid1c of each). As expected, two distinct clades, one made up of Jarid1d genes and one made up of Jarid1c genes, show that these two loci have evolved separately since before the origins of the species examined here (Fig. 1). This topology also suggests that if gene conversion has occurred, it has not included this portion of these genes.

The tree topology built using only Jarid1d and Jarid1c is unresolved in several clades. To obtain a well-supported and completely resolved topology for use in ancestral reconstruction, maximum parsimony and maximum likelihood outgroup-rooted trees were built using sequences from six nuclear genes including Jarid1d and Jarid1c (Tucker et al. 2005). These trees are identical to each other, but this topology differs from the Jarid1d- and Jarid1cspecific tree from the first analysis in two ways. The branches that were previously unresolved are now resolved, and the relationship of Hylomyscus and Mastomys is changed (Fig. 2). This tree topology was converted to an unrooted topology for the analysis.

Ancestral reconstruction and counting changes. Different methods of ancestral reconstruction using the nuclear gene tree topology (Fig. 2) led to different results. ACCTRAN, DELTRAN, and Bayesian-based reconstructions differ in a few sites at most nodes. The results of both types of Bayesian reconstructions, Poisson and JTT, are the 


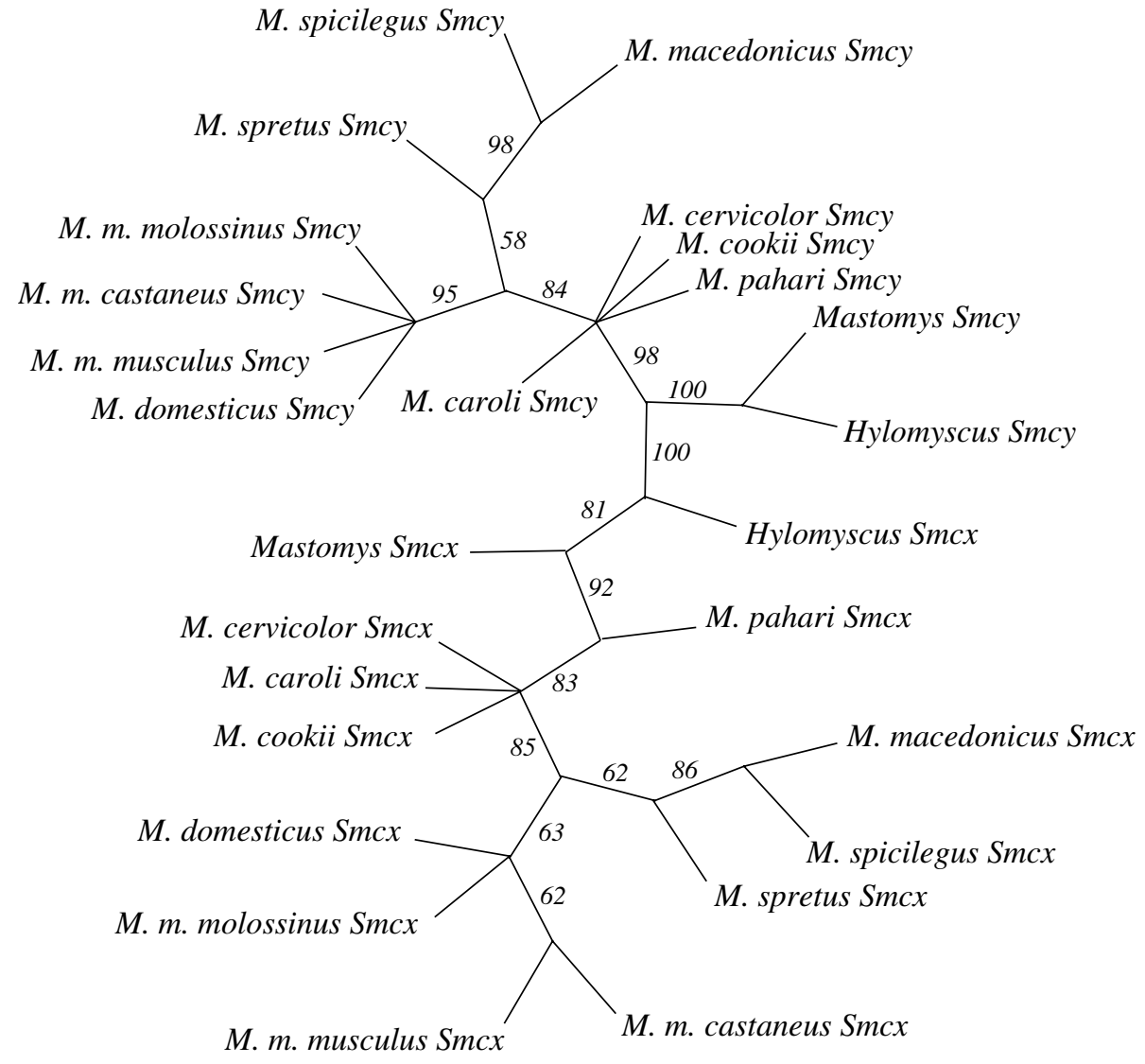

Fig. 1. Jarid1d/Jarid1c maximum parsimony majority rule consensus tree for members of the genus Mus and members of a putative sister group, Hylomyscus alleni and Mastomys hildebrandtii. Numbers are bootstrap values. same, and each node has a posterior probability of $\geq 0.99$ for protein reconstruction $\times$ DNA reconstruction.

Numbers of replacement and silent substitutions within the genus Mus were counted on each reconstructed tree. Using the ACCTRAN reconstructions, Jarid1c has 5 replacement and 14 silent changes. Jarid1d has 22 replacement and 17 silent changes (Table 1). The exact numbers of substitutions differ with the different methods of reconstructing ancestral sequences but in each case the results are similar: There are more replacement substitutions relative to silent substitutions in Jarid1d than in Jarid1c. For each reconstruction method, Fisher's exact test shows a significant difference in the numbers of synonymous and nonsynonymous substitutions between Jarid1d and Jarid1c $(p<0.05)$ (Table 1). When replacement and silent substitutions of the most distantly related taxa, Hylomyscus and Mastomys, are included with those of Mus, the difference is significant only when the DELTRAN reconstructions are used. This disparity in results can be traced to the long branch between the Hylomyscus/Mastomys clade and the Mus clade, where uncorrected distances may not be appropriate measures.

\section{Discussion}

Higher-than-average $\mathrm{dN} / \mathrm{dS}$ are found in the mammalian sex-determining gene Sry (Tucker and Lundrigan 1993; Whitfield et al. 1993; reviewed by O'Neill and O'Neill 1999; Jansa et al. 2003), and in $Z f_{y}$ (Tucker et al. 2003). The dN/dS of Sry and $Z f_{y}$ are also higher than the $\mathrm{dN} / \mathrm{dS}$ of their X chromosomal gametologs, Sox3 and $Z f_{X}$ (Pamilo and Waugh O'Neill 1997; Wyckoff et al. 2002; Tucker et al. 2003) (Table 2). The results of previous research on Sry and Zfy are compatible with either a Y chromosomal effect on the efficiency of purifying selection or alternate explanations invoking locus-specific effects such as adaptive evolution or relaxed functional constraints (Tucker and Lundrigan 1993; Whitfield et al. 1993; Hurst 1994a, b; Lee et al. 1995; Tucker et al. 2003). If unique properties of the $\mathrm{Y}$ chromosome are responsible for the patterns of evolution in Sry and $Z f_{y}$, a similar pattern should be seen in other $\mathrm{Y}$ chromosomal genes, including Jarid1d.

However, it is possible that the relatively high $\mathrm{dN} / \mathrm{dS}$ of Sry and $Z f y$ is a result of natural selection on gene functions that have changed compared with their counterparts on the X chromosome. Generally, 
Table 1. Comparison of replacement and silent substitutions found in Jarid1d and Jarid1c

\begin{tabular}{|c|c|c|c|c|c|c|c|c|c|c|c|}
\hline \multirow{2}{*}{$\begin{array}{l}\text { Ancestral reconstruction } \\
\text { method }\end{array}$} & \multicolumn{5}{|c|}{ Jarid1d } & \multicolumn{5}{|c|}{ Jarid1c } & \multirow{2}{*}{$\begin{array}{l}\text { Fisher's exact } \\
\text { test }(P)^{\mathrm{f}}\end{array}$} \\
\hline & $n^{\mathrm{a}}$ & $N^{\mathrm{b}}$ & $s^{\mathrm{c}}$ & $S^{\mathrm{d}}$ & $d N / d S$ & $n$ & $N$ & $s$ & $S$ & $d N / d S$ & \\
\hline ACCTRAN & 22 & 505.36 & 17 & 151.36 & 0.366 & 5 & 505.64 & 14 & 151.36 & 0.126 & 0.03 \\
\hline DELTRAN & 22 & 505.64 & 19 & 151.36 & 0.347 & 16.5 & 485.84 & 41.5 & 141.16 & 0.132 & 0.01 \\
\hline Poisson and $\mathrm{JTT}^{\mathrm{e}}$ & 89 & 505.45 & 66 & 151.39 & 0.404 & 26.7 & 485.66 & 43.25 & 171.34 & 0.283 & 0.01 \\
\hline
\end{tabular}

${ }^{\mathrm{a}}$ Number of replacement substitutions counted in Mus clade.

${ }^{\mathrm{b}}$ Average potential replacement substitutions per branch in Mus clade.

${ }^{\mathrm{c}}$ Number of silent substitutions counted in Mus clade.

${ }^{\mathrm{d} A v e r a g e}$ potential silent substitutions per branch in Mus clade.

${ }^{\mathrm{e}}$ Poisson and JTT Bayesian reconstruction results were the same.

${ }^{\mathrm{f}}$ Test of significance of difference in number of nonsynonymous (Jarid1 $d_{n}$, Jarid $\left.1 c_{n}\right)$ and synonymous $\left(\operatorname{Jarid}_{1} d_{s}, \operatorname{Jarid}_{1} c_{s}\right)$ substitutions.

genes expressed in many tissues (such as $Z f_{X}$ ) have a lower $\mathrm{dN} / \mathrm{dS}$ than genes expressed in few tissues (such as $Z f y$ ), and genes expressed in the brain (such as Sox3) have significantly lower dN than genes expressed in other tissues (such as Sry) (Duret and Mouchiroud 2000). Thus, Zfy and Sry may have relatively high $\mathrm{dN} / \mathrm{d} S$ because of positive selection for new functions or because of their limited patterns of expression instead of their locations on the $\mathrm{Y}$ chromosome. Jarid1c and Jarid1d were chosen for this study because the difference in their $\mathrm{dN} / \mathrm{dS}$ is less likely to be affected by gene function (Agulnik et al. 1999; but see Jensen et al. 2005) or expression patterns (Agulnik et al. 1994a, b).

Neither Jarid1c nor Jarid1d has diverged rapidly. Along some branches of the Mus tree (Fig. 2), there are no substitutions in either gene. Although this did not substantially affect our conclusions, it does indicate that tests designed for less-diverged sequences are preferable. One such test (Zhang et al. 1997) showed that Jarid1d has significantly more replacement substitutions than Jarid1c has. This result is consistent with a three-taxon study of Jar$i d 1 c / d$ in human, mouse, and horse, in which the median $\mathrm{dN} / \mathrm{dS}$ was 0.17 for Jarid1d and 0.02 for Jarid1c (Agulnik et al. 1997). Though the exact numbers reported by Agulnik et al. (1997) differ from those that we find using other methods, this indicates that there are relatively more replacement substitutions in Jarid1d in both closely related and distantly related taxa.
Although the $\mathrm{dN} / \mathrm{dS}$ of Jarid1d on the Y chromosome is higher than the $\mathrm{dN} / \mathrm{dS}$ of Jarid1c on the $\mathrm{X}$ chromosome $(0.40$ and 0.28 , respectively, when calculated using the Poisson method), they are similar relative to other X-Y gene pairs. This is not necessarily what would be expected based on the length of time that Jarid1d and Jarid1c have been diverging from one another. The mammalian $\mathrm{X}$ and $\mathrm{Y}$ likely diverged from each other in a series of steps, separated by time. The present-day signatures of these steps are strata, defined by the synonymous distance between pairs of gametologs on the $\mathrm{X}$ and $\mathrm{Y}$ (Lahn and Page 1999). The gene pairs Sox3/Sry, Zfx/y, and Jarid1c/d diverged at different times as shown by the $\mathrm{X}$ chromosomal strata of the X chromosomal members of the pairs (Lahn and Page 1999; Sandstedt and Tucker 2004). Since Jarid1c is either in X chromosomal stratum 1 or 2 , it has been diverging from Jarid1d longer than $Z f_{X}[\mathrm{dN} / \mathrm{dS}=0.04$ (Tucker et al. 2003)], a stratum 3 gene, has been diverging from $Z f y$ $[\mathrm{dN} / \mathrm{dS}=0.38$ (Tucker et al. 2003)]. The relatively subtle difference between Jarid1d's and Jarid1c's dN/ dS may be due to greater functional constraints on Jarid1d than on other $\mathrm{Y}$ chromosomal genes or to lesser functional constraint on Jarid1c than on other $\mathrm{X}$ chromosomal genes (in the part of the gene studied) (Table 2). Data from Wyckoff et al. (2002) also show that the difference in $\mathrm{dN} / \mathrm{dS}$ between Jarid1d and Jarid1c is smaller than the difference between several other $\mathrm{X}$-Y gene pairs in pairwise comparisons of mouse genes with human genes.

Table 2. Comparison of $\mathrm{dN} / \mathrm{dS}$ of gametologous gene pairs in the genus Mus

\begin{tabular}{lcll}
\hline Gene & Chromosome & $d N / d S^{\mathrm{a}}$ & Source \\
\hline Jarid1d & $\mathrm{Y}$ & 0.404 & This paper \\
Jarid1c & $\mathrm{X}$ & 0.283 & This paper \\
Sry & $\mathrm{Y}$ & 0.478 & (Jansa et al. 2003) \\
Sox3 & $\mathrm{X}$ & $0.088^{\mathrm{b}}$ & (Pamilo and Waugh O'Neill 1997) \\
$Z f_{y}$ & $\mathrm{Y}$ & 0.38 & (Tucker et al. 2003) \\
$Z f_{X}$ & $\mathrm{X}$ & 0.04 & (Tucker et al. 2003) \\
\hline
\end{tabular}

${ }^{a}$ Calculated using various methods.

${ }^{b}$ From human-mouse comparison. 


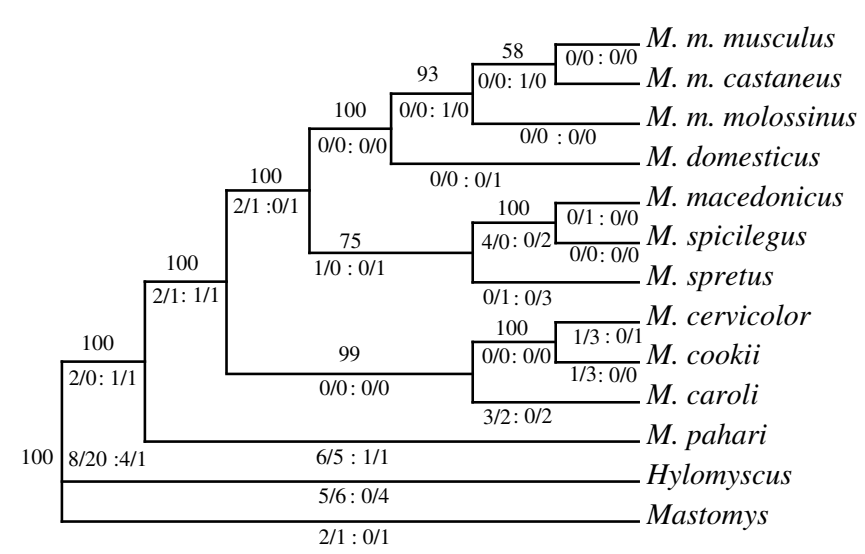

Fig. 2. Single most parsimonious tree using Jarid1d, Jarid1c, Sry, B2m, Zp-3, and Tcp-1 (Tucker et al. 2005) for the genus Mus. Rooted with outgroups Hylomyscus alleni and Mastomys hildebrandtii. Numbers above the branches are bootstrap values. Numbers below the branches are Jarid $1 d_{n} /$ Jarid1d $d_{s}$ Jarid $1 c_{n} /$ Jarid1c $c_{s}$ reconstructed using the ACCTRAN method.

Another implication of the position of these gene pairs in strata, which diverged at different times, is that the elevated $\mathrm{dN} / \mathrm{dS}$ of $\mathrm{Y}$ chromosomal genes cannot be completely attributed to positive selection for new functions. If $\mathrm{Y}$ chromosomal genes gained new functions soon after they stopped recombining with their X chromosomal gametologs, they would have many nonsynonymous changes during that time, but few later after their new functions were obtained (Wyckoff et al. 2002). That pattern has not been seen in mouse $\mathrm{X}-\mathrm{Y}$ gene pairs from strata 1 through 3; instead a high nonsynonymous substitution rate continues along recent branches within the genus Mus, not just in ancestral portions of the mammalian tree (current study; Tucker and Lundrigan 1993; Tucker et al. 2003).

Sequence from the autosomal locus Jarid1b from Mus musculus corresponding to the sequenced region of Jarid1c and Jarid1d is available in GenBank (accession No. BC048180). The sequence of Mus musculus Jarid1a was not alignable with this region. About 120 amino acids could be aligned with confidence between the $J a r i d 1 b / c / d$ genes. There are no nonsynonymous changes in Jarid1c in this region and five nonsynonymous changes in Jarid1d. No nonsynonymous substitutions of Jarid1d change amino acids that are conserved in both Jarid1b and Jarid1c. This may indicate that Jarid1d remains evolutionarily constrained by specific, important codons, perhaps because of a need for dosage compensation relative to Jarid1c.

Genes that survive on the Y chromosome either have acquired critical male-specific functions or are required for equivalent dosage relative to their $\mathrm{X}$ - linked gametologs. Jarid1d is functionally constrained and ubiquitously expressed, while its Xlinked gametolog Jarid1c escapes $\mathrm{X}$ inactivation to some degree in many tissues. We find that there are more nonsynonymous changes in Jarid1d than in Jaird1c, but it is a subtle difference relative to other $\mathrm{X} / \mathrm{Y}$ gene pairs. Together these factors suggest that dosage requirements have had a greater impact on the evolution of Jarid1d than male-specific functions.

While it is possible that non-Y-specific effects may be responsible for the pattern of evolution of Jarid1d, the results of this study, combined with the results of studies of other Y-linked genes in these same taxa (Tucker and Lundrigan 1993; Jansa et al. 2003; Tucker et al. 2003), strongly suggest that there is a Y chromosomal effect on gene evolution in the genus Mus. The $\mathrm{Y}$ chromosomal effect occurs for genes with different expression patterns, found in one or many copies. The $\mathrm{X}$ chromosomal gametologs of these genes differ in their $\mathrm{X}$ inactivation patterns and their strata. Taken together, these observations suggest that (1) a higher $\mathrm{dN} / \mathrm{dS}$ is a constant feature of $\mathrm{Y}$ chromosomal gene evolution, which is not limited to a burst of nonsynonymous change soon after X-Y gene pair divergence and (2) it occurs in dissimilar genes. The results reported herein were obtained from gametologs assumed to be functionally equivalent, using closely related taxa, and in a well-supported phylogenetic context. Indeed, they are in accord with and strengthen the results of a pairwise comparison of six X-Y gametologs in distantly related mammals in which the $\mathrm{dN} / \mathrm{dS}$ was always higher in the $\mathrm{Y}$ chromosomal gene (Wyckoff et al. 2002).

\section{Acknowledgments}

The authors thank Jianzhi Zhang for his comments on an earlier draft of this work. This work was supported by NIH grant EY12994 and NSF grant DEB9209950.

\section{References}

1. Agulnik AI, Mitchell MJ, Lerner JL, Woods DR, Bishop CE (1994a) A mouse Y-chromosome gene encoded by a region essential for spermatogenesis and expression of male-specific minor histocompatibility antigens. Hum Mol Genet 3, 873-878

2. Agulnik AI, Mitchell MJ, Mattei M-G, Borsani G, Avner PA, et al. (1994b) A novel X gene with a widely transcribed Y-linked homologue escapes X-inactivation in mouse and human. Hum Mol Genet 3, 879-884

3. Agulnik AI, Bishop CE, Lerner JL, Agulnik SI, Solovyev VV (1997) Analysis of mutation rates in the 
SMCY/SMCX genes shows that mammalian evolution is male driven. Mamm Genome 8, 134-138

4. Agulnik AI, Longepied G, Ty MT, Bishop CE, Mitchell M (1999) Mouse H-Y encoding Smcy gene and its X chromosomal homolog Smcx. Mamm Genome 10, 926-929

5. Bull JJ (1983) Evolution of sex determining mechanisms (Menlo Park, CA: Benjamin/Cummings)

6. Catzeflis FM, Denys C (1992) The African Nannomys (Muridae) - an early offshoot from the Mus lineage-evidence from scnDNA hybridization experiments and compared morphology. Isr J Zool 38, 219-231

7. Ceplitis H, Ellegren H (2004) Adaptive molecular evolution of HINTW, a female-specific gene in birds. Mol Biol Evol 21, 249-254

8. Charlesworth B, Charlesworth D (2000) The degeneration of Y chromosomes. Philos Trans R Soc Lond B Biol Sci 355, 1563-1572

9. Chevret P, Granjon L, Duplantier JM, Denys C, Catzeflis FM (1994) Molecular phylogeny of the Praomys complex (Rodentia, Murinae)—a study based on DNA/ DNA hybridization experiments. Zool J Linn Soc 112, 425-442

10. Disteche CM (1999) Escapees on the X chromosome. Proc Natl Acad Sci USA 96, 14180-14182

11. Duret L, Mouchiroud D (2000) Determinants of substitution rates in mammalian genes: expression pattern affects selection intensity but not mutation rate. Mol Biol Evol 17, 68-74

12. Fridolfsson A-K, Cheng H, Copeland NG, Jenkins NA, Liu H-C, et al. (1998) Evolution of the avian sex chromosomes from an ancestral pair of autosomes. Proc Natl Acad Sci USA 95, 8147-8152

13. Garcia-Moreno J, Mindell DP (2000) Rooting a phylogeny with homologous genes on opposite sex chromosomes (gametologs): a case study using avian $C H D$. Mol Biol Evol 17, 1826-1832

14. Hurst LD (1994a) Embryonic growth and the evolution of the mammalian $\mathrm{Y}$ chromosome I.: The $\mathrm{Y}$ as an attractor for selfish growth factors. Heredity 73, $223-232$

15. Hurst LD (1994b) Embryonic growth and the evolution of the mammalian Y chromosome II: Suppression of the selfish Y-linked growth factors may explain escape from $\mathrm{X}$-inactivation and rapid evolution of Sry. Heredity 73, 233-243

16. Jansa SA, Lundrigan BL, Tucker PK (2003) Tests for positive selection on immune and reproductive genes in closely related species of the murine genus Mus. J Mol Evol 56, 294-307

17. Jansa SA, Weksler M (2004) Phylogeny of muroid rodents: relationships within and among major lineages as determined by IRBP gene sequences. Mol Phylogenet Evol 31, 256-276

18. Jegalian K, Page DC (1998) A proposed path by which genes common to mammalian $\mathrm{X}$ and $\mathrm{Y}$ chromosomes evolve to become $\mathrm{X}$ inactivated. Nature 394, 776-780

19. Jensen LR, Amende M, Gurok U, Moser B, Gimmel V, et al. (2005) Mutations in the JARID1C gene, which is involved in transcriptional regulation and chromatin remodeling, cause X-linked mental retardation. Am J Hum Genet 76, 227-236

20. Kumar S, Tamura Y, Jakobsen IB, Nei M (2001) MEGA 2: Molecular evolutionary genetics analysis software. Bioinformatics 17, 1244-1245

21. Lahn BT, Page DC (1997) Functional coherence of the human Y-chromosome. Science 278, 675-680

22. Lahn BT, Page DC (1999) Four evolutionary strata on the human X chromosome. Science 286, 964-967

23. Lahn BT, Pearson NM, Jegalian K (2001) The human Y chromosome, in the light of evolution. Nat Rev Genet 2, 207-216

24. Lee YH, Ota T, Vacquier VD (1995) Positive selection is a general phenomenon in the evolution of abalone sperm lysin. Mol Biol Evol 12, 231-238

25. Lundrigan BL, Jansa SA, Tucker PK (2002) Phylogenetic relationships in the genus Mus, based on maternally, paternally, and biparentally inherited characters. Syst Biol 51, 410-431

26. Maddison D, Maddison W (2000) MacClade 4: Analysis of Phylogeny and Character Evolution (Sunderland, MA: Sinauer Associates)

27. Negrutiu I, Vyskot B, Barbacar N, Georgiev S, Moneger F (2001) Dioecious plants. A key to the early events of sex chromosome evolution. Plant Physiol 127, $1418-1424$

28. Nei M, Gojobori J (1986) Simple methods for estimating the number of synonymous and nonsynonymous nucleotide substitutions. Mol Biol Evol 3, 418-426

29. O’Neill MJ, O'Neill RJW (1999) Whatever happened to SRY? Cell Mol Life Sci 56, 883-893

30. Pamilo P, Waugh O'Neill RJ (1997) Evolution of the Sry genes. Mol Biol Evol 14, 49-55

31. Patsialou A, Wilsker D, Moran E (2005) DNA-binding properties of ARID family proteins. Nucleic Acids Res 33, 66-80

32. Rice WR (1996) Evolution of the Y sex chromosome in animals. BioScience 46, 331-343

33. Saiki RK, Scharf S, Faloona F, Mullis KB, Horn GT, et al. (1985) Enzymatic amplification of $\beta$-globin genomic sequences and restriction site analysis for diagnosis of sickle cell anemia. Science 230, 1350-1254

34. Sandstedt SA, Tucker PK (2004) Evolutionary strata on the mouse $\mathrm{X}$ chromosome correspond to strata on the human X chromosome. Genome Res 14, 267-272

35. Scott DM, Ehrmann IE, Ellis PS, Agulnik AI, Bishop CE, et al. (1995) Identification of a mouse male-specific transplantation antigen, H-Y. Nature 376, 695-698

36. Sheardown S, Norris D, Fisher A, Brockdorff N (1996) The mouse Smcx gene exhibits developmental and tissue specific variation in degree of escape from $\mathrm{X}$ inactivation. Hum Mol Genet 5, 1355-1360

37. Steinemann M, Steinemann S (1998) Enigma of $Y$ chromosome degeneration: neo-Y and neo-X chromosomes of Drosophila miranda a model for sex chromosome evolution. Genetica 102-103, 409-420

38. Swofford DL (2002) PAUP* Phylogenetic Analysis Using Parsimony (* and Other Methods) (Sunderland, MA: Sinauer Associates) 
39. Takeuchi T, Yamazaki Y, Katohfukui Y, Tsuchiya R, Kondo S, et al. (1995) Gene trap capture of a novel mouse gene, Jumonji, required for neural-tube formation. Genes Devel 9, 1211-1222

40. Thompson JD, Gibson TJ, Plewniak F, Jeanmougin F, Higgins DG (1997) The CLUSTAL X windows interface: flexible strategies for multiple sequence alignment aided by quality analysis tools. Nucleic Acids Res 25, 4876-4888

41. Toder R, Wakefield MJ, Graves JAM (2000) The minimal mammalian $\mathrm{Y}$ chromosome - the marsupial $\mathrm{Y}$ as a model system. Cytogenet Cell Genet 91, 285-292

42. Tucker PK, Lundrigan BL (1993) Rapid evolution of the sex determining locus in Old World mice and rats. Nature 364, 715-717

43. Tucker PK, Adkins RM, Rest JS (2003) Differential rates of evolution for the $Z F Y$-related zinc finger genes, $Z f_{y}, Z f_{X}$ and $Z f a$ in the mouse genus Mus. Mol Biol Evol 20, 999-1005

44. Tucker PK, Sandstedt SA, Lundrigan BL (2005) Phylogenetic relationships in the subgenus Mus (genus Mus, family Muridae, subfamily Murinae): examining gene trees and species trees. Biol J Linn Soc 84, 653-662

45. Wang W, Meadows LR, den Haan JMM, Sherman NE, Chen Y, et al. (1995) Human H-Y: a male-specific histocompatibility antigen derived from the SMCY protein. Science 269, 1588-1590
46. Whitfield LS, Lovell-Badge R, Goodfellow PN (1993) Rapid sequence evolution of the mammalian sexdetermining gene SRY. Nature 364, 713-715

47. Wu J, Ellison J, Salido E, Yen P, Mohandas T, et al. (1994a) Isolation and characterization of XE169, a novel human gene that escapes X-inactivation. Hum Mol Genet 3, 153-160

48. Wu J, Salido EC, Yen PH, Mohandas TK, Heng HHQ, et al. (1994b) The murine Xe169 gene escapes X-inactivation like its human homologue. Nat Genet 7, 491-496

49. Wyckoff GJ, Li J, Wu C-I (2002) Molecular evolution of functional genes on the mammalian Y chromosome. Mol Biol Evol 19, 1633-1636

50. Xu J, Burgoyne PS, Arnold AP (2002) Sex differences in sex chromosome gene expression in mouse brain. Hum Mol Genet 11, 1409-1419

51. Zhang J (2004) Evolution of DMY, a newly emergent male sex-determination gene of medaka fish. Genetics $166,1887-1895$

52. Zhang J, Nei M (1997) Accuracies of ancestral amino acid sequences inferred by the parsimony, likelihood, and distance methods. J Mol Evol 44, S139-S146

53. Zhang J, Kumar S, Nei M (1997) Small-sample tests of episodic adaptive evolution: A case study of primate lysozymes. Mol Biol Evol 14, 1335-1338

54. Zhang J, Rosenberg HF, Nei M (1998) Positive Darwinian selection after gene duplication in primate ribonuclease genes. Proc Natl Acad Sci USA 95, 3708-3713 